\title{
Volumetric and Ultrasonic Velocity Studies of Urea and Thiourea in Aqueous Solution
}

\author{
ROKSANA KHATUN, RAJIA SULTANA and RANJIT K. NATH* \\ Department of Chemistry, Faculty of Engineering \& Technology, Chittagong University of \\ Engineering \& Technology (CUET), Chittagong-4349, Bangladesh. \\ *Corresponding author E-mail: rkn_chem@yahoo.com \\ http://dx.doi.org/10.13005/ojc/340407
}

(Received: January 02, 2018; Accepted: August 06, 2018)

\begin{abstract}
The observations on the anomalous behavior of urea and the comparison between urea and thiourea in aqueous solutions have been examined by volumetric and ultrasonic sound velocity techniques at different temperature $(298.15,303.15,308.15,313.15,318.15$ and $323.15 \mathrm{~K}$ ), atmospheric pressure by using a high accuracy vibrating U-tube digital density and ultrasonic sound velocity analyzer. The apparent molar volume $\left(\varphi_{v}\right) \&$ apparent molar adiabatic compressibility $\left(\varphi_{k}\right)$ have been calculated from experimental density and ultrasonic sound velocity data respectively and limiting apparent molar volume $\left(\varphi^{v}{ }_{0}\right)$, limiting apparent molar adiabatic compressibility $\left(\varphi_{0}^{k}\right)$ have been evaluated from apparent molar volume vs. molality plot as intercept. Apparent molar expansibility $\left(\varphi_{E}\right)$ was determined from apparent molar volume and hydration number $\left(n_{H}\right)$ from adiabatic compressibility. The results show very interesting information about strong solute-solvent \& solute-solute interactions, and also elaborate the structure making or breaking behavior in the solution mixtures.
\end{abstract}

Keywords: Urea, Thiourea, Apparent molar volume, Expansibility, Sound velocity, Hydration number.

\section{INTRODUCTION}

The physico-chemical interaction between various essential molecules in a living organism and cosolutes are very important. Urea is highly active compound in a variety of biological functions in our body and has been referred as protein denaturing agent. Urea provides a significant role in the metabolism of compounds having nitrogen by animals and the highest amount of substance contain nitrogen in the urine of mammals. Body use it in various functions; the most important is nitrogen excretion. Further, urea is an essential basic material for the chemical industry. Thiourea is structurally similar to urea except that the oxygen atom in urea is replaced by a sulfur atom, although the properties of urea \& thiourea differ considerably. Thiourea is also a valuable reagent in organic synthesis processes and employed as a source of sulfide. Substituted thioureas are beneficial catalysts for many organic

This is an Open Access article licensed under a Creative Commons Attribution-Non Commercial-Share Alike 4.0 International License (https://creativecommons.org/licenses/by-nc-sa/4.0/), which permits unrestricted Non Commercial use, distribution and reproduction in any medium, provided the original work is properly cited. 
synthesis reactions. Other industrial applications of thiourea such as production of flame retardant resins, vulcanization accelerators, auxiliary agent in diazo paper \& light-sensitive photocopy paper and used to tone silver-gelatin photographic prints are also useful applications.

The volumetric and ultrasonic sound velocity data deliver valuable information about the interactions such as solute-solute, solute-solvent etc. Maximum researcher reported that ${ }^{1,2}$ urea behave as structure breaker in water. Some researcher reported that urea perform as a structure maker ${ }^{3}$ in water. On the other hand, it has been shown that by thermo-chemical \& NMR studies of urea has no basically net effect on the structure of water ${ }^{4}$. So, the interaction of urea with water is not yet clearly discussed. For clear observations about the urea effect on water, we study the various parameters of molecular interaction in aqueous urea solutions through volumetric and ultrasonic measurements and also studied the comparison between urea and thiourea. The ultrasonic sound velocity and density measurements ${ }^{5,6}$ and their derived parameter such as, apparent molar volume, limiting apparent molar volume, apparent molar expansibility, adiabatic compressibility, apparent molar adiabatic compressibility and hydration number ${ }^{7,8}$ find wide applications in characterizing the physico-chemical behavior of solution mixture.

\section{EXPERIMENTAL}

\section{Materials}

Urea (Purity declared by supplier, mass fraction $>0.995 \%$ with molar mass $0.06006 \mathrm{~kg} \cdot \mathrm{mol}^{-1}$ ) and thiourea (Purity confirmed by supplier, mass fraction $>0.995 \%$ with molar mass $0.07612 \mathrm{~kg} \cdot \mathrm{mol}^{-1}$ ) was collected from Loba Chemie Pvt. Ltd, India.

\section{Measurement of Density and Ultasonic Sound Velocity}

The solutions were prepared by using freshly redistilled and degassed water (specific conductance $<10^{-6} \mathrm{~S} \mathrm{~cm}^{-1}$ ). The solutions were prepared (in molality) by weighing on a balance (Mettler Toledo, B204-S, Switzerland) having an accuracy of $\pm 0.0001 \mathrm{~g}$. The densities $(\rho)$ and ultrasonic sound velocity $(u)$ of the solutions were instantly and automatically measured using a density and ultrasonic sound velocity analyzer (DSA 5000,
Anton Paar, Austria). A density check or an adjustment of air/water was done at $20^{\circ} \mathrm{C}$ by using triply distilled, degassed water and dry air at atmospheric pressure. Before measurements, the analyzer was calibrated with redistilled \& degassed water in the selection experimental temperature range. Both the density and ultrasonic sound velocity are very sensitive to temperature, thus it was organized to $\pm 1 \cdot 10^{-3} \mathrm{~K}$ by a built-in Peltier device. The sensitivity of the instrument relates to a precision in density and ultrasonic sound velocity measurements of $1 \cdot 10^{-3} \mathrm{~kg} \mathrm{~m}^{-3}$ and $1 \cdot 10^{-2}$ $\mathrm{m} \mathrm{s}^{-1} \cdot 9$

\section{RESULT AND DISCUSSIONS}

\section{Apparent molar volume \& apparent molar adiabatic compressibility}

The apparent molar volume $(\varphi v)$ \& apparent molar adiabatic compressibility $(\varphi k)$ are very valuable parameters in the understanding of interactions between solute-solvent and solute-solute. The density and ultrasonic sound velocity data are used to calculate $\varphi \mathrm{v}, \beta \mathrm{s}$ and $\varphi \mathrm{k}$ by using the relations ${ }^{2}(1),(2) \&(3)$.

$$
\begin{aligned}
& \phi_{v}=\frac{M_{2}}{\rho}-\frac{\rho-\rho_{\mathrm{o}}}{m \rho \rho_{\mathrm{o}}} \\
& \beta_{s}=\frac{1}{\rho u^{2}} \\
& \phi_{k}=\frac{\beta_{s} \rho_{0}-\rho \beta_{s}^{0}}{m \rho \rho_{0}}+\frac{\beta_{s} M_{2}}{\rho}
\end{aligned}
$$

Where $\mathrm{m} /\left(\mathrm{mol} \mathrm{kg}^{-1}\right)$ is the molality of urea/thiourea in aqueous solutions, $\rho /\left(\mathrm{kg} \mathrm{m}^{-3}\right)$ is the density of urea/thiourea solution, $\rho 0 /\left(\mathrm{kg} \mathrm{m}^{-3}\right)$ is the density of solvent and $\mathrm{M}_{2} /\left(\mathrm{kg} \mathrm{mol}^{-1}\right)$ is the molar mass of urea/thiourea.

The experimental density $(\rho)$ and ultrasonic sound velocity $(u)$ of aqueous solution of urea \& thiourea are represented in Table 1. \& Table 2. as a function of molality urea \& thiourea and temperature. The $\varphi_{v}$ values are represented in Table 1. The $\varphi_{v}$ values of urea and thiourea in water at different concentration are also graphically presented in Fig. 1(a) and Fig. 1(b) respectively. From the data, it is clearly observed that $\varphi v$ values of urea \& thiourea increase with both the increase in concentration and increase in temperature. The usual explanation is 
that the interactions of solute species follows through the destructive overlap of their hydration spheres 10 . Urea $\left(\mathrm{H}_{2} \mathrm{~N}-\mathrm{CO}-\mathrm{NH}_{2}\right)$ and thiourea $\left(\mathrm{H}_{2} \mathrm{~N}-\mathrm{CS}-\mathrm{NH}_{2}\right)$ molecules contain $-\mathrm{NH}_{2},-\mathrm{CO}$ and $-\mathrm{CS}$ groups which are hydrophilic groups. So, interaction between solute and water molecules complete through hydrophilic hydration. The interaction of two hydrophilic hydration co-spheres releases some water molecules from the hydration sphere to the bulk results of an increase in volume with an

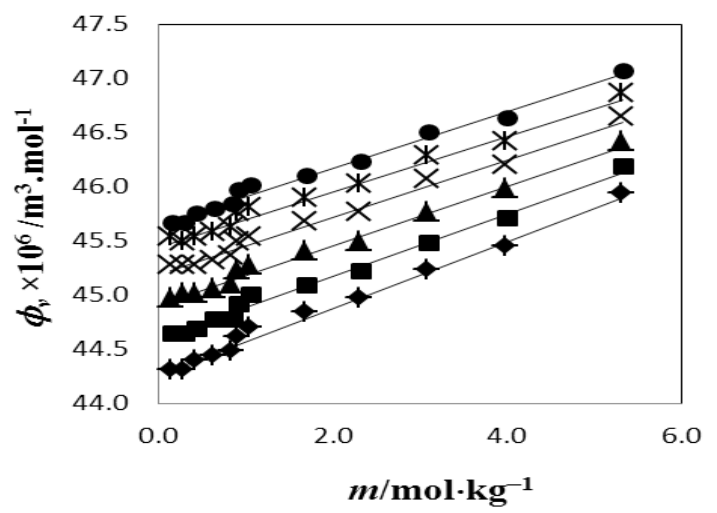

(a) increase in the concentration of urea \& thiourea. As the temperature is increased several water molecules from the hydration co-sphere relaxes from the cosphere to the bulk due to thermal agitation thereby increasing the $\varphi v$. Thiourea contain a less hydrophilicity sulfer group, in presence of these group $\left(-\mathrm{CS} \ldots . \mathrm{H}_{2} \mathrm{O}\right)$ hydrogen bond in thiourea is weak and less compact hydration than $\left(-\mathrm{CO} \ldots . \mathrm{H}_{2} \mathrm{O}\right)$ hydrogen bond in urea molecule. For this reason $\varphi_{v}$ values are greater in thiourea.

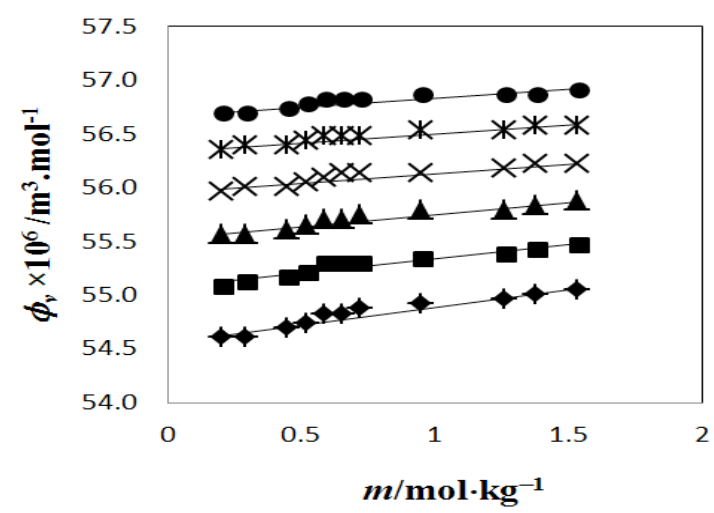

(b)

Fig. 1. Apparent molar volume of (a) urea (b) thiourea in water as a function of molality at different temperatures; - -298.15K, !-303.15K, $\Delta-308.15 \mathrm{~K}, \square-313.15 \mathrm{~K}$, $, 3-318.15 \mathrm{~K}, \bullet-323.15 \mathrm{~K}$

The calculated $\varphi_{k}$ values of urea and thiourea in water at different temperatures are represented in Table 2 . The $\varphi_{k}$ values of urea and thiourea in water are graphically presented in Fig. 2(c) and Fig. 2(d) respectively. The $\varphi_{k}$ values are gradually changes and become negative when the temperature and concentration of urea and thiourea are low. The solute-solute interactions involve the interactions between hydrophilic-hydrophilic hydration spheres. These interactions between the co-spheres of hydrophilic groups result the relaxation

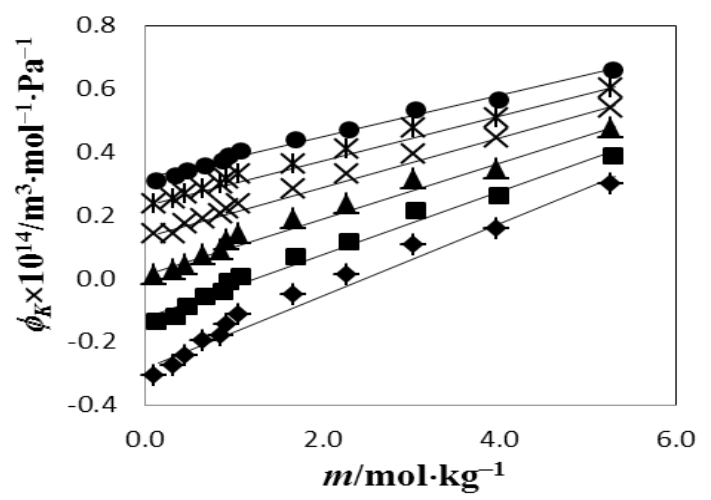

(c) of water molecules from hydrophilic zone to bulk. The water molecule relaxation from hydrophilic zone to bulk results the positive change in $\varphi_{k}$. The hydrophilic effect always produce overall positive effect on $\varphi_{k}$ causing an increase in apparent molar adiabatic compressibility with the concentration of solutes. More water molecules relaxes from hydrophilic zone to bulk in thiourea solutions due to the week $\left(-\mathrm{CS} \ldots . \mathrm{H}_{2} \mathrm{O}\right)$ hydrogen bond, which makes the more positive change in $\varphi_{k}$ values for thiourea compare with urea solution.

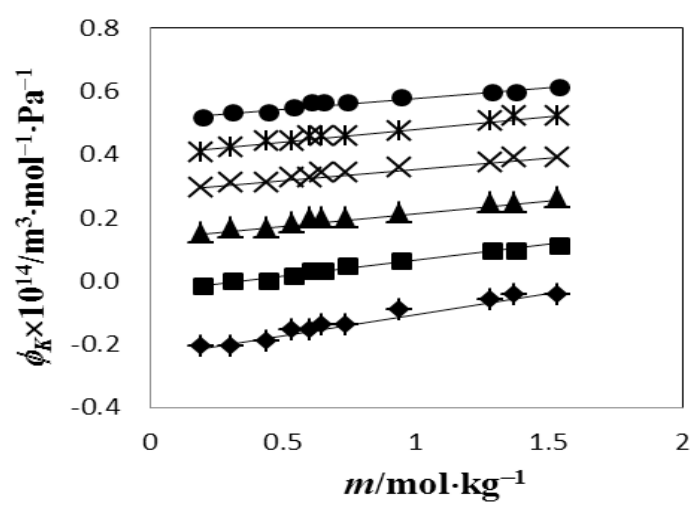

(d)

Fig. 2. Apparent molar adiabatic compressibility of (c) urea (d) thiourea in water as a function of molality at different temperatures; $\bullet-298.15 \mathrm{~K}$, !-303.15K, $\Delta-308.15 \mathrm{~K}, \square-313.15 \mathrm{~K},{ }^{-}-318.15 \mathrm{~K}, \bullet-323.15 \mathrm{~K}$ 
Table 1: Densities, $\rho / \mathrm{kg} \cdot \mathrm{m}^{-3}$, apparent molar volume, $\varphi_{\mathrm{v}} /\left(10^{-6} \bullet \mathrm{m}^{3 \bullet} \mathrm{mol}^{-1}\right)$ and limiting apparent molar volume, $\varphi_{\mathrm{v}}{ }^{0} /\left(10^{-6} \bullet \mathrm{m}^{3} \bullet \mathrm{mol}^{-1}\right)$ of urea and thiourea in aqueous solutions at 298.15, 303.15, $308.15,313.15,318.15$ and $323.15 \mathrm{~K}$ respectively

\begin{tabular}{|c|c|c|c|c|c|c|c|}
\hline $\begin{array}{l}\text { Molality (m) } \\
\text { mol•kg-1 }\end{array}$ & $\begin{array}{l}\text { Urea } \\
\qquad \rho\end{array}$ & $\varphi_{\mathrm{v}}$ & $\varphi_{v}{ }^{0}$ & 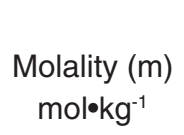 & $\begin{array}{c}\text { Thiourea } \\
\rho\end{array}$ & $\varphi_{\mathrm{v}}$ & $\varphi_{\mathrm{v}}{ }^{0}$ \\
\hline \multicolumn{8}{|c|}{$\mathrm{T}=298.15 \mathrm{~K}$} \\
\hline 0.00000 & 997.044 & & & 0.00000 & 997.044 & & \\
\hline 0.14333 & 999.300 & 44.30 & & 0.19984 & 1001.321 & 54.58 & \\
\hline 0.30767 & 1001.848 & 44.32 & & 0.30720 & 1003.571 & 54.62 & \\
\hline 0.44066 & 1003.860 & 44.38 & & 0.45193 & 1006.550 & 54.67 & \\
\hline 0.63874 & 1006.801 & 44.44 & & 0.53601 & 1008.230 & 54.74 & \\
\hline 0.89270 & 1010.494 & 44.48 & & 0.59753 & 1009.432 & 54.81 & \\
\hline 0.95956 & 1011.340 & 44.61 & 44.26 & 0.65270 & 1010.538 & 54.81 & 54.55 \\
\hline 1.08983 & 1013.123 & 44.68 & & 0.73880 & 1012.226 & 54.84 & \\
\hline 1.68355 & 1021.051 & 44.81 & & 0.95090 & 1016.312 & 54.90 & \\
\hline 2.31850 & 1028.957 & 44.95 & & 1.27989 & 1022.472 & 54.96 & \\
\hline 3.08261 & 1037.401 & 45.24 & & 1.38780 & 1024.420 & 54.99 & \\
\hline 4.03605 & 1047.202 & 45.45 & & 1.53355 & 1027.004 & 55.04 & \\
\hline 5.32037 & 1057.912 & 45.93 & & & & & \\
\hline \multicolumn{8}{|c|}{$\mathrm{T}=303.15 \mathrm{~K}$} \\
\hline 0.00000 & 995.644 & & & 0.00000 & 995.644 & & \\
\hline 0.14333 & 997.857 & 44.65 & & 0.19984 & 999.830 & 55.09 & \\
\hline 0.30767 & 1000.355 & 44.67 & & 0.30720 & 1002.034 & 55.12 & \\
\hline 0.44066 & 1002.335 & 44.71 & & 0.45193 & 1004.947 & 55.17 & \\
\hline 0.63874 & 1005.224 & 44.76 & & 0.53601 & 1006.608 & 55.21 & \\
\hline 0.89270 & 1008.856 & 44.80 & & 0.59753 & 1007.790 & 55.27 & \\
\hline 0.95956 & 1009.686 & 44.93 & & 0.65270 & 1008.870 & 55.28 & 55.07 \\
\hline 1.08983 & 1011.441 & 44.99 & 44.60 & 0.73880 & 1010.532 & 55.30 & \\
\hline 1.68355 & 1019.246 & 45.11 & & 0.95090 & 1014.546 & 55.35 & \\
\hline 2.31850 & 1027.029 & 45.24 & & 1.27989 & 1020.592 & 55.40 & \\
\hline 3.08261 & 1035.396 & 45.50 & & 1.38780 & 1022.522 & 55.42 & \\
\hline 4.03605 & 1045.102 & 45.69 & & 1.53355 & 1025.074 & 55.45 & \\
\hline 5.32037 & 1055.592 & 46.18 & & & & & \\
\hline \multicolumn{8}{|c|}{$\mathrm{T}=308.15 \mathrm{~K}$} \\
\hline 0.00000 & 994.025 & & & 0.00000 & 994.025 & & \\
\hline 0.14333 & 996.201 & 44.96 & & 0.19984 & 998.135 & 55.53 & \\
\hline 0.30767 & 998.652 & 44.99 & & 0.30720 & 1000.295 & 55.57 & \\
\hline 0.44066 & 1000.606 & 45.01 & & 0.45193 & 1003.162 & 55.60 & \\
\hline 0.63874 & 1003.454 & 45.05 & & 0.53601 & 1004.793 & 55.64 & \\
\hline 0.89270 & 1007.028 & 45.09 & 44.90 & 0.59753 & 1005.956 & 55.70 & 55.53 \\
\hline 0.95956 & 1007.847 & 45.21 & & 0.65270 & 1007.018 & 55.70 & \\
\hline 1.08983 & 1009.576 & 45.27 & & 0.73880 & 1008.654 & 55.72 & \\
\hline 1.68355 & 1017.263 & 45.39 & & 0.95090 & 1012.612 & 55.75 & \\
\hline
\end{tabular}




\begin{tabular}{|c|c|c|c|c|c|c|c|}
\hline 2.31850 & 1024.941 & 45.51 & & 1.27989 & 1018.572 & 55.79 & \\
\hline 3.08261 & 1033.177 & 45.76 & & 1.38780 & 1020.457 & 55.82 & \\
\hline 4.03605 & 1042.735 & 45.95 & & 1.53355 & 1022.968 & 55.85 & \\
\hline 5.32037 & 1053.154 & 46.41 & & & & & \\
\hline \multicolumn{8}{|c|}{$\mathrm{T}=313.15 \mathrm{~K}$} \\
\hline 0.00000 & 992.207 & & & 0.00000 & 992.207 & & \\
\hline 0.14333 & 994.35 & 45.25 & & 0.19984 & 996.249 & 55.94 & \\
\hline 0.30767 & 996.765 & 45.28 & & 0.30720 & 998.375 & 55.98 & \\
\hline 0.44066 & 998.692 & 45.29 & & 0.45193 & 1001.197 & 56.00 & \\
\hline 0.63874 & 1001.5 & 45.33 & & 0.53601 & 1002.802 & 56.04 & \\
\hline 0.89270 & 1005.023 & 45.36 & & 0.59753 & 1003.946 & 56.10 & \\
\hline 0.95956 & 1005.832 & 45.48 & 45.19 & 0.65270 & 1004.991 & 56.10 & 55.95 \\
\hline 1.08983 & 1007.537 & 45.54 & & 0.73880 & 1006.604 & 56.11 & \\
\hline 1.68355 & 1015.11 & 45.66 & & 0.95090 & 1010.512 & 56.13 & \\
\hline 2.31850 & 1022.707 & 45.76 & & 1.27989 & 1016.392 & 56.15 & \\
\hline 3.08261 & 1030.743 & 46.05 & & 1.38780 & 1018.241 & 56.19 & \\
\hline 4.03605 & 1040.312 & 46.19 & & 1.53355 & 1020.744 & 56.20 & \\
\hline 5.32037 & 1050.604 & 46.64 & & & & & \\
\hline \multicolumn{8}{|c|}{$\mathrm{T}=318.15 \mathrm{~K}$} \\
\hline 0.00000 & 990.204 & & & 0.00000 & 990.204 & & \\
\hline 0.14333 & 992.318 & 45.51 & & 0.19984 & 994.184 & 56.33 & \\
\hline 0.30767 & 994.712 & 45.50 & & 0.30720 & 996.277 & 56.37 & \\
\hline 0.44066 & 996.605 & 45.54 & & 0.45193 & 999.061 & 56.38 & \\
\hline 0.63874 & 999.378 & 45.58 & & 0.53601 & 1000.644 & 56.41 & \\
\hline 0.89270 & 1002.858 & 45.61 & & 0.59753 & 1001.772 & 56.47 & \\
\hline 0.95956 & 1003.658 & 45.73 & & 0.65270 & 1002.800 & 56.47 & \\
\hline 1.08983 & 1005.341 & 45.79 & 45.45 & 0.73880 & 1004.391 & 56.48 & 56.33 \\
\hline 1.68355 & 1012.837 & 45.89 & & 0.95090 & 1008.234 & 56.51 & \\
\hline 2.31850 & 1020.334 & 46.00 & & 1.27989 & 1014.024 & 56.53 & \\
\hline 3.08261 & 1028.285 & 46.28 & & 1.38780 & 1015.872 & 56.54 & \\
\hline 4.03605 & 1037.802 & 46.40 & & 1.53355 & 1018.319 & 56.57 & \\
\hline 5.32037 & 1047.947 & 46.85 & & & & & \\
\hline \multicolumn{8}{|c|}{$\mathrm{T}=323.15 \mathrm{~K}$} \\
\hline 0.00000 & 988.019 & & & 0.00000 & 988.019 & & \\
\hline 0.14333 & 990.121 & 45.67 & & 0.19984 & 991.950 & 56.67 & \\
\hline 0.30767 & 992.495 & 45.68 & & 0.30720 & 994.018 & 56.69 & \\
\hline 0.44066 & 994.359 & 45.76 & & 0.45193 & 996.765 & 56.72 & \\
\hline 0.63874 & 997.099 & 45.81 & & 0.53601 & 998.321 & 56.76 & \\
\hline 0.89270 & 1000.539 & 45.84 & & 0.59753 & 999.430 & 56.82 & \\
\hline 0.95956 & 1001.333 & 45.96 & 45.65 & 0.65270 & 1000.454 & 56.81 & 56.67 \\
\hline 1.08983 & 1002.997 & 46.01 & & 0.73880 & 1002.029 & 56.81 & \\
\hline 1.68355 & 1010.417 & 46.11 & & 0.95090 & 1005.821 & 56.84 & \\
\hline 2.31850 & 1017.833 & 46.22 & & 1.27989 & 1011.534 & 56.87 & \\
\hline 3.08261 & 1025.71 & 46.49 & & 1.38780 & 1013.354 & 56.88 & \\
\hline 4.03605 & 1035.012 & 46.64 & & 1.53355 & 1015.759 & 56.91 & \\
\hline 5.32037 & 1045.189 & 47.06 & & & & & \\
\hline
\end{tabular}


Table 2: Ultrasonic velocity, $\mathrm{u} /\left({\left.\mathrm{m} . \mathrm{s}^{-1}\right)}\right.$ adiabatic compressibility, $\beta_{\mathrm{s}} /\left(10^{-10} \bullet \mathrm{Pa}^{-1}\right)$ apparent molar adiabatic compressibility, $\varphi_{k} /\left(10^{-14} \bullet \mathrm{m}^{3} \bullet \mathrm{mol}^{-1} . \mathrm{Pa}-1\right)$ limiting apparent molar volume, $\varphi_{k}{ }^{0}(10-$ $\left.{ }^{14} \cdot \mathrm{m}^{3} \bullet \mathrm{mol}^{-1} \cdot \mathrm{Pa}^{-1}\right)$ and hydration number, $\mathrm{nH}$ of urea and thiourea in aqueous solutions at 298.15, $303.15,308.15,313.15,318.15$ and $323.15 \mathrm{~K}$ respectively

\begin{tabular}{|c|c|c|c|c|c|c|c|c|c|c|c|}
\hline \multirow{2}{*}{$\begin{array}{l}\text { Molality (m } \\
\text { moløkg }^{-1}\end{array}$} & \multirow{3}{*}{ n) } & \multicolumn{3}{|c|}{ Urea } & \multirow[b]{2}{*}{$\mathrm{nH}$} & \multirow[b]{2}{*}{ 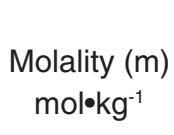 } & \multicolumn{3}{|c|}{ Thiourea } & \multirow{3}{*}{$\varphi_{\mathrm{k}}^{0}$} & \multirow{3}{*}{$\mathrm{n}_{\mathrm{H}}$} \\
\hline & & $\beta_{\mathrm{s}}$ & $\varphi_{\mathrm{k}}$ & $\varphi_{\mathrm{k}}^{0}$ & & & $\mathrm{u}$ & $\beta_{\mathrm{s}}$ & $\varphi_{\mathrm{k}}$ & & \\
\hline \multicolumn{9}{|c|}{$\mathrm{T}=298.15 \mathrm{~K}$} & & & \\
\hline 0.00000 & 1496.79 & 4.477 & & & & 0.00000 & 1496.79 & 4.477 & & & \\
\hline 0.14333 & 1500.58 & 4.444 & -0.315 & & 2.8261 & 0.19984 & 1502.41 & 4.424 & -0.215 & & 3.2544 \\
\hline 0.30767 & 1504.74 & 4.408 & -0.277 & & 2.7597 & 0.30720 & 1505.40 & 4.397 & -0.205 & & 3.2255 \\
\hline 0.44066 & 1507.98 & 4.381 & -0.244 & & 2.7075 & 0.45193 & 1509.40 & 4.361 & -0.192 & & 3.1870 \\
\hline 0.63874 & 1512.63 & 4.341 & -0.203 & & 2.6374 & 0.53601 & 1511.54 & 4.341 & -0.162 & & 3.1405 \\
\hline 0.89270 & 1518.65 & 4.291 & -0.179 & & 2.5834 & 0.59753 & 1513.27 & 4.326 & -0.159 & & 3.1303 \\
\hline 0.95956 & 1519.94 & 4.280 & -0.147 & & 2.5439 & 0.65270 & 1514.56 & 4.314 & -0.138 & & 3.0957 \\
\hline 1.08983 & 1522.84 & 4.256 & -0.128 & -0.281 & 2.5107 & 0.73880 & 1516.97 & 4.293 & -0.139 & -0.240 & 3.0854 \\
\hline 1.68355 & 1535.46 & 4.154 & -0.061 & & 2.3785 & 0.95090 & 1522.17 & 4.247 & -0.096 & & 3.0031 \\
\hline 2.31850 & 1547.98 & 4.056 & 0.002 & & 2.2534 & 1.27989 & 1530.47 & 4.175 & -0.067 & & 2.9219 \\
\hline 3.08261 & 1560.60 & 3.958 & 0.102 & & 2.0886 & 1.38780 & 1533.07 & 4.153 & -0.053 & & 2.8920 \\
\hline 4.03605 & 1577.33 & 3.838 & 0.158 & & 1.9621 & 1.53355 & 1536.73 & 4.123 & -0.043 & & 2.8612 \\
\hline 5.32037 & 1592.88 & 3.725 & 0.295 & & 1.7523 & & & & & & \\
\hline \multicolumn{12}{|c|}{$\mathrm{T}=303.15 \mathrm{~K}$} \\
\hline 0.00000 & 1509.18 & 4.410 & & & & 0.00000 & 1509.18 & 4.410 & & & \\
\hline 0.14333 & 1500.58 & 4.380 & -0.139 & & 2.6269 & 0.19984 & 1514.31 & 4.362 & -0.018 & & 3.0364 \\
\hline 0.30767 & 1504.74 & 4.347 & -0.118 & & 2.5835 & 0.30720 & 1516.99 & 4.337 & 0.000 & & 2.9988 \\
\hline 0.44066 & 1507.98 & 4.321 & -0.091 & & 2.5369 & 0.45193 & 1520.73 & 4.303 & -0.003 & & 2.9810 \\
\hline 0.63874 & 1512.63 & 4.284 & -0.061 & & 2.4818 & 0.53601 & 1522.72 & 4.284 & 0.018 & & 2.9440 \\
\hline 0.89270 & 1518.65 & 4.238 & -0.035 & & 2.4251 & 0.59753 & 1524.25 & 4.271 & 0.026 & & 2.9279 \\
\hline 0.95956 & 1519.94 & 4.227 & -0.009 & -0.123 & 2.3934 & 0.65270 & 1525.50 & 4.259 & 0.040 & & 2.9035 \\
\hline 1.08983 & 1522.84 & 4.205 & 0.009 & & 2.3619 & 0.73880 & 1527.65 & 4.240 & 0.042 & -0.036 & \\
\hline \multicolumn{12}{|l|}{2.8886} \\
\hline 1.68355 & 1535.46 & 4.110 & 0.068 & & 2.2407 & 0.95090 & 1532.65 & 4.196 & 0.066 & & 2.8311 \\
\hline 2.31850 & 1547.98 & 4.018 & 0.122 & & 2.1271 & 1.27989 & 1540.15 & 4.131 & 0.099 & & 2.7469 \\
\hline 3.08261 & 1560.60 & 3.926 & 0.211 & & 1.9757 & 1.38780 & 1542.65 & 4.110 & 0.105 & & 2.7253 \\
\hline 4.03605 & 1577.33 & 3.813 & 0.259 & & 1.8599 & 1.53355 & 1545.99 & 4.082 & 0.114 & & 2.6957 \\
\hline 5.32037 & 1592.88 & 3.707 & 0.385 & & 1.6639 & & & & & & \\
\hline \multicolumn{12}{|c|}{$\mathrm{T}=308.15 \mathrm{~K}$} \\
\hline 0.00000 & 1519.88 & 4.355 & & & & 0.00000 & 1519.88 & 4.355 & & & \\
\hline 0.14333 & 1523.06 & 4.327 & 0.006 & & 2.4600 & 0.19984 & 1524.60 & 4.310 & 0.141 & & 2.8563 \\
\hline 0.30767 & 1526.64 & 4.296 & 0.021 & & 2.4248 & 0.30720 & 1527.07 & 4.287 & 0.157 & & 2.8219 \\
\hline 0.44066 & 1529.40 & 4.273 & 0.043 & & 2.3835 & 0.45193 & 1530.44 & 4.256 & 0.162 & & 2.7948 \\
\hline 0.63874 & 1533.44 & 4.238 & 0.069 & & 2.3343 & 0.53601 & 1532.29 & 4.239 & 0.178 & & 2.7649 \\
\hline 0.89270 & 1538.54 & 4.195 & 0.090 & 0.014 & 2.2846 & 0.59753 & 1533.64 & 4.226 & 0.190 & 0.134 & 2.7439 \\
\hline 0.95956 & 1539.73 & 4.185 & 0.113 & & 2.2569 & 0.65270 & 1534.83 & 4.215 & 0.198 & & 2.7270 \\
\hline 1.08983 & 1542.22 & 4.165 & 0.128 & & 2.2288 & 0.73880 & 1536.78 & 4.198 & 0.201 & & 2.7115 \\
\hline
\end{tabular}




\begin{tabular}{|c|c|c|c|c|c|c|c|c|c|c|c|}
\hline 1.68355 & 1553.07 & 4.076 & 0.180 & & 2.1173 & 0.95090 & 1541.48 & 4.156 & 0.213 & & 2.6684 \\
\hline 2.31850 & 1563.94 & 3.989 & 0.227 & & 2.0137 & 1.27989 & 1548.48 & 4.094 & 0.237 & & 2.5964 \\
\hline 3.08261 & 1574.89 & 3.902 & 0.309 & & 1.8731 & 1.38780 & 1550.78 & 4.075 & 0.243 & & 2.5754 \\
\hline 4.03605 & 1589.51 & 3.796 & 0.351 & & 1.7662 & 1.53355 & 1553.85 & 4.049 & 0.252 & & 2.5473 \\
\hline 32037 & 1603.18 & 3.694 & 0.466 & & 1.5838 & & & & & & \\
\hline \multicolumn{12}{|c|}{$\mathrm{T}=313.15 \mathrm{~K}$} \\
\hline 00000 & 1528.95 & 4.311 & & & & 0.00000 & 1528.95 & 4.311 & & & \\
\hline 0.14333 & 1531.88 & 4.286 & 0.130 & & 2.3135 & 0.19984 & 1533.27 & 4.270 & 0.288 & & 2.6859 \\
\hline 0.30767 & 1535.18 & 4.257 & 0.143 & & 2.2816 & 0.30720 & 1535.53 & 4.248 & 0.302 & & 2.6543 \\
\hline 0.44066 & 1537.75 & 4.234 & 0.159 & & 2.2481 & 0.45193 & 1538.62 & 4.219 & 0.306 & & 2.6303 \\
\hline 0.63874 & 1541.51 & 4.202 & 0.180 & & 2.2055 & 0.53601 & 1540.32 & 4.203 & 0.319 & & 2.6035 \\
\hline 0.89270 & 1546.26 & 4.162 & 0.197 & & 2.1615 & 0.59753 & 1541.56 & 4.191 & 0.330 & & 2.5843 \\
\hline 0.95956 & 1547.34 & 4.152 & 0.220 & & 2.1338 & 0.65270 & 1542.66 & 4.181 & 0.336 & & 2.5697 \\
\hline 1.08983 & 1549.66 & 4.133 & 0.233 & 0.133 & 2.1084 & 0.73880 & 1544.46 & 4.165 & 0.337 & 0.280 & 2.5567 \\
\hline 1.68355 & 1559.84 & 4.049 & 0.277 & & 2.0094 & 0.95090 & 1548.77 & 4.126 & 0.347 & & 2.5172 \\
\hline 2.31850 & 1569.92 & 3.967 & 0.320 & & 1.9121 & 1.27989 & 1554.96 & 4.069 & 0.378 & & 2.4386 \\
\hline 3.08261 & 1580.16 & 3.886 & 0.397 & & 1.7800 & 1.38780 & 1557.16 & 4.050 & 0.380 & & 2.4241 \\
\hline 4.03605 & 1593.84 & 3.784 & 0.431 & & 1.6825 & 1.53355 & 1560.01 & 4.026 & 0.384 & & 2.4011 \\
\hline 2037 & 1606.69 & 3.687 & 0.537 & & 1.5116 & & & & & & \\
\hline \multicolumn{12}{|c|}{$\mathrm{T}=318.15 \mathrm{~K}$} \\
\hline .00000 & 1536.45 & 4.278 & & & & 0.00000 & 1536.45 & 4.278 & & & \\
\hline 0.14333 & 1539.16 & 4.254 & 0.236 & & 2.1865 & 0.19984 & 1540.44 & 4.239 & 0.408 & & 2.5454 \\
\hline 0.30767 & 1542.23 & 4.227 & 0.241 & & 2.1631 & 0.30720 & 1542.51 & 4.219 & 0.424 & & 2.5119 \\
\hline 0.44066 & 1544.62 & 4.206 & 0.258 & & 2.1314 & 0.45193 & 1545.33 & 4.191 & 0.430 & & 2.4860 \\
\hline 0.63874 & 1548.14 & 4.175 & 0.274 & & 2.0950 & 0.53601 & 1546.92 & 4.176 & 0.439 & & 2.4651 \\
\hline 0.89270 & 1552.56 & 4.137 & 0.290 & & 2.0538 & 0.59753 & 1548.02 & 4.166 & 0.453 & & 2.4423 \\
\hline 0.95956 & 1553.57 & 4.128 & 0.311 & & 2.0281 & 0.65270 & 1549.08 & 4.156 & 0.454 & & 2.4341 \\
\hline 1.08983 & 1555.73 & 4.110 & 0.323 & 0.233 & 2.0043 & 0.73880 & 1550.72 & 4.140 & 0.456 & 0.398 & 2.4203 \\
\hline 1.68355 & 1565.26 & 4.030 & 0.361 & & 1.9141 & 0.95090 & 1554.52 & 4.104 & 0.475 & & 2.3710 \\
\hline 2.31850 & 1574.65 & 3.953 & 0.401 & & 1.8222 & 1.27989 & 1560.09 & 4.052 & 0.506 & & 2.2945 \\
\hline 3.08261 & 1584.25 & 3.875 & 0.472 & & 1.6989 & 1.38780 & 1561.99 & 4.035 & 0.511 & & 2.2771 \\
\hline 4.03605 & 1597.09 & 3.778 & 0.501 & & 1.6086 & 1.53355 & 1564.60 & 4.012 & 0.515 & & 2.2564 \\
\hline 5.32037 & 1609.19 & 3.685 & 0.601 & & 1.4472 & & & & & & \\
\hline \multicolumn{12}{|c|}{$\mathrm{T}=323.15 \mathrm{~K}$} \\
\hline 0.00000 & 1542.50 & 4.254 & & & & 0.00000 & 1542.50 & 4.254 & & & \\
\hline 0.14333 & 1545.03 & 4.231 & 0.314 & & 2.0885 & 0.19984 & 1546.20 & 4.217 & 0.510 & & 2.4253 \\
\hline 0.30767 & 1547.83 & 4.206 & 0.332 & & 2.0502 & 0.30720 & 1548.10 & 4.198 & 0.528 & & 2.3896 \\
\hline 0.44066 & 1550.13 & 4.185 & 0.339 & & 2.0340 & 0.45193 & 1550.70 & 4.172 & 0.534 & & 2.3639 \\
\hline 0.63874 & 1553.41 & 4.156 & 0.355 & & 1.9984 & 0.53601 & 1552.15 & 4.158 & 0.546 & & 2.3411 \\
\hline 0.89270 & 1557.53 & 4.120 & 0.370 & & 1.9591 & 0.59753 & 1553.15 & 4.148 & 0.561 & & 2.3177 \\
\hline 0.95956 & 1558.49 & 4.112 & 0.389 & & 1.9360 & 0.65270 & 1554.15 & 4.138 & 0.558 & & 2.3134 \\
\hline 1.08983 & 1560.50 & 4.094 & 0.401 & 0.317 & 1.9130 & 0.73880 & 1555.65 & 4.124 & 0.561 & 0.507 & 2.2996 \\
\hline 1.68355 & 1569.41 & 4.018 & 0.436 & & 1.8286 & 0.95090 & 1559.15 & 4.090 & 0.578 & & 2.2532 \\
\hline 2.31850 & 1578.21 & 3.945 & 0.473 & & 1.7426 & 1.27989 & 1564.44 & 4.039 & 0.600 & & 2.1899 \\
\hline 3.08261 & 1587.22 & 3.870 & 0.538 & & 1.6267 & 1.38780 & 1566.25 & 4.023 & 0.602 & & 2.1755 \\
\hline 4.03605 & 1599.29 & 3.777 & 0.567 & & 1.5405 & 1.53355 & 1568.70 & 4.001 & 0.606 & & 2.1566 \\
\hline
\end{tabular}


Limiting apparent molar volume \& limiting apparent molar adiabatic compressibility

The limiting apparent molar volume $\left(\varphi_{v}{ }^{0}\right)$ and limiting apparent molar adiabatic compressibility $\left(\varphi_{k}{ }^{0}\right)$ values were attained by least squares method to the equation (4) and (5),

$$
\begin{aligned}
& \phi_{v}=\phi_{v}^{0}+S_{v} m \\
& \phi_{k}=\phi_{k}^{0}+S_{k} m
\end{aligned}
$$

The $\varphi_{v}{ }^{0}$ and $\varphi_{k}{ }^{0}$ values of urea and thiourea in water are reported in Table 1. and Table 2. respectively against the concentration of urea \& thiourea at various temperatures. It is clearly observed that the values of $\varphi_{v}{ }^{0}$ are positive and increase with increase in temperature, which indicates that there exists strong interactions between solute-solvent that are more suitable at higher temperatures. The $\varphi_{v}{ }^{0}$ increase with increase in temperature may be owing to the following facts: (i) at higher temperature due to the increasing thermal energy of water molecules, the hydrophilic water molecules is relaxed from the interaction regions of $-\mathrm{NH}_{2},-\mathrm{CO}$ and $-\mathrm{CS}$ groups of urea, thiourea molecules results of a positive volume change \& (ii) the interactions between water and water decreases with increase in temperature giving rise a very small negative change in volume. The linear increasing change of $\varphi_{v}$ with molality shows that the interactions between urea and water increase with the increase in concentration of urea at the experimental temperatures. Similar nature was stated previously by concentration dependence studies of apparent molar volume of aqueous solutions of urea by Stokes 11 .

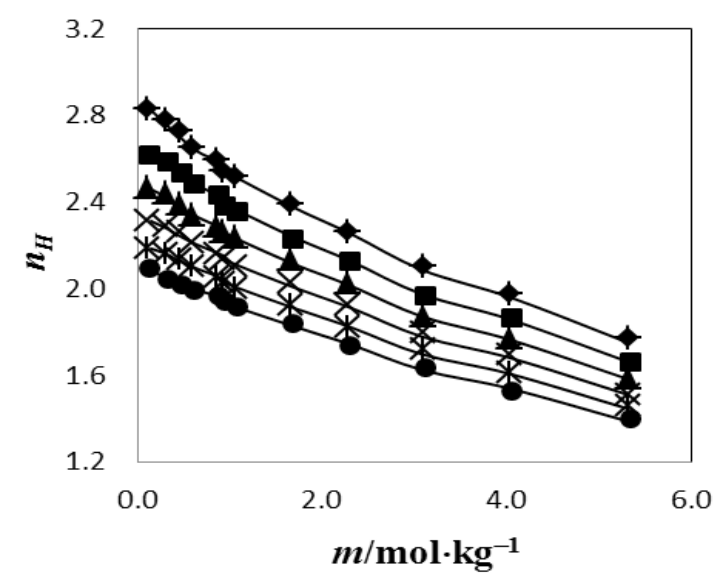

It was observed that the values of $\varphi_{k}{ }^{0}$ are negative at low temperatures \& the magnitudes of $\varphi_{k}{ }^{0}$ values increase with increase in temperature and become positive in magnitude at higher experimental temperatures. The negative values of $\varphi_{k}^{0}$ specify that the water molecules surrounding urea \& thiourea are less compressible than that are present in bulk medium and increase in magnitude or become positive at elevated temperature, which may be recognized to the melting of rigid hydration structures around the urea and thiourea molecule. The water molecules exist in the monomeric form are more compressible.

\section{Hydration number}

The hydration number of amino acid was calculated using equation ${ }^{12}(6)$

$$
n_{H}=\frac{n_{W}}{n_{S}}\left(1-\frac{\beta_{S}}{\beta_{S}^{0}}\right)
$$

where $n_{w}$ is the number of moles of water $\mathrm{n}_{\mathrm{s}}$ is the number of moles of solute,

$\beta_{s} / \mathrm{Pa}^{-1}$ is the adiabatic compressibility of aqueous solution and

$\beta_{\mathrm{s}}{ }^{0} / \mathrm{Pa}^{-1}$ is the adiabatic compressibility of urea and thiourea in aqueous solution.

The hydration number of urea and thiourea calculated by above equation are listed in Table 2 . The $n_{H}$ values of urea and thiourea in water are also graphically represented in Fig. 3(e) and Fig. 3(f) respectively. From the data, it is observed that $\mathrm{nH}$ values of urea \& thiourea decrease with increase in the temperature and with increase in concentration of urea and thiourea.

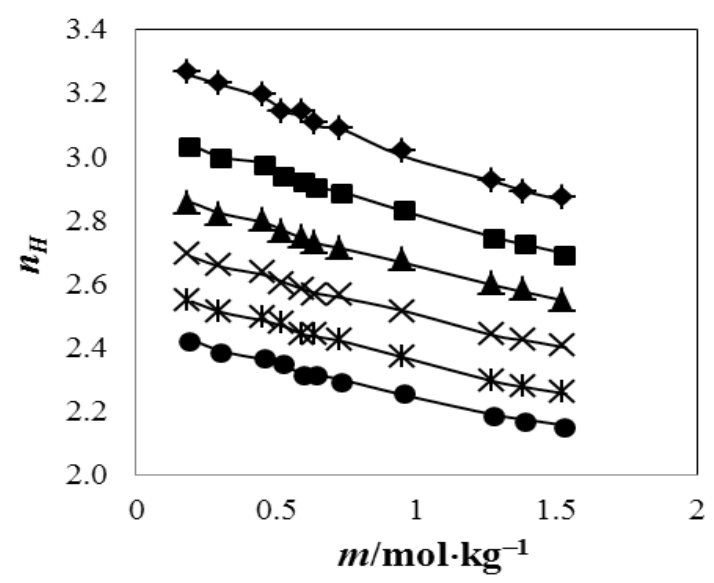

Fig. 3. Hydration number of (e) urea ( $f$ ) thiourea in water as a function of molality at different temp.; $-298.15 \mathrm{~K}$, !-303.15K, $\Delta-308.15 \mathrm{~K}, \square-313.15 \mathrm{~K}$, ж- 318.15K, $\bullet-323.15 \mathrm{~K}$ 
Table 3: Fitting parameters of equation 7 for $\varphi_{v}$, limiting apparent molar expansibilities $\left(\varphi_{E}{ }^{0}\right)$ and values of the Hepler's Constant $\left(\partial^{2} \varphi_{\mathrm{v}}{ }^{\%} / \partial \mathrm{T}_{2}\right)_{\mathrm{p}}$ of urea and thiourea in aqueous solutions at $298.15,303.15,308.15,313.15,318.15$ and $323.15 \mathrm{~K}$ respectively

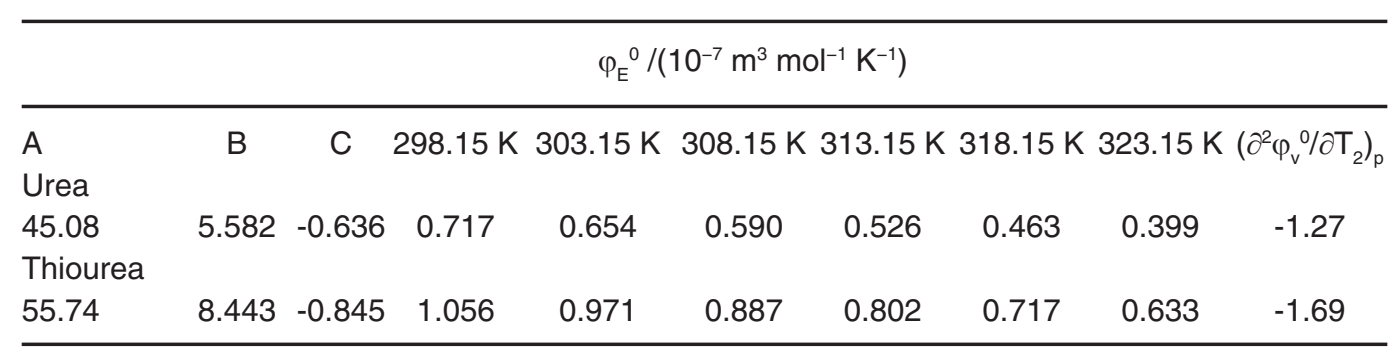

The decrease in hydration number with the increase in urea and thiourea molality is attributed to the removal of $\mathrm{H}_{2} \mathrm{O}$ molecules from the hydration sphere due to the overlap of cospheres of urea and thiourea molecules. As the temperature is increased some $\mathrm{H}_{2} \mathrm{O}$ molecules from hydration cosphere relaxes to the bulk due to thermal agitation thereby decreasing the hydration number. $n_{H}$ of thiourea is more than the urea due to the bigger sulphur atom which is less hydrophilic than oxygen atom in urea molecule. Due to the increasing hydrophobicity behavior of thiourea, more hydration number observes for thiourea than urea in solution.

\section{Limiting Apparent Molar Expansibility}

The $\varphi_{v}{ }^{0}$ values are highly sensitive to temperature ${ }^{13}$, and can be retrogression against the temp. using following equation ${ }^{14-16}$,

$\varphi_{\mathrm{v}}^{0}=\mathrm{A}+\mathrm{B}\left(\mathrm{T}-\mathrm{T}_{\mathrm{m}}\right)+\mathrm{C}\left(\mathrm{T}-\mathrm{T}_{\mathrm{M}}\right) 2$

Where, $T$ is the temperature in Kelvin, $T_{m}$ is mean value of the studied temp. (here $T_{m}=310.65$ $K)$. The value of coefficients $A, B$ \& $C$ were measured through polynomial fits and summarized in Table 3. The limiting partial molar expansibility $\left(\varphi_{E}{ }^{0}\right)$, noted as a partial derivative of limiting partial molar volume with respect to temp., can be measured using the $B$ \& C parameters described in above relation.

$\varphi_{E}{ }^{0}=\left(\partial \varphi_{v}{ }^{0}\right) \partial T_{p}=B+2 C\left(T-T_{m}\right)$

Calculated values of $\varphi_{\mathrm{E}}{ }^{0}$ are shown in Table 3. with the fitting parameters of eq. 7. The Hepler's constant $\left(\partial^{2} \varphi_{v}{ }^{0} / \partial T^{2}\right) p$, provides detailed information on the hydration interaction in terms of structure making and breaking capacity of the solute, which can be obtained from equation (9) $\left(\mathrm{d} \varphi_{\mathrm{E}}{ }^{0}\right) /(\mathrm{dT}=)\left(\partial^{2} \varphi_{\mathrm{v}}{ }^{0}\right)\left(\partial \mathrm{T}^{2}\right)_{\mathrm{p}}=2 \mathrm{C}$

Hepler ${ }^{17-18}$ argued that a positive value of $\left(\partial^{2} \varphi_{v}{ }_{v} / \partial T_{2}\right)_{p}$ is associated with structure making nature, whereas a negative value of $\left(\partial^{2} \varphi_{v}{ }^{0} / \partial T_{2}{ }^{p}\right.$ is associated with a structure braking nature. It is apparent that the values of $\left(\partial^{2} \varphi_{v}{ }_{v} / \partial T_{2}\right)_{p}$ are negative for urea and thiourea in aqueous solution are negative and $\left(\partial^{2} \varphi_{v}{ }^{0} / \partial T_{2}\right)_{p}$ value for thiourea is more negative than urea solution. Urea and thiourea solutions are attributed to the coordination of water molecules around urea and thiourea molecules through hydrophilic hydration. When the temperature is increased, the interactions between solute molecules become significant, and the hydrated water molecules around the hydrophilic groups are relaxed to bulk, and hence, urea and thiourea shows structure-breaking behavior. Probably due to bigger atomic size of sulfur, more interaction occurs between thiourea molecules and shows more structure breaking behavior. In hydration number observation, it's observed that hydration number of thiourea is higher than urea, this is due to the more interaction of water molecule detach from hydration sphere to bulk results of more structure breaking behavior of thiourea.

\section{CONCLUSION}

The densities and ultrasonic velocity of urea and thiourea in water were calculated at different temperatures \& atmospheric pressure. From the measurements, apparent molar volumes $(\varphi \vee)$ apparent molar adiabatic compressibility ( $\varphi \mathrm{k})$ limiting apparent molar volume $\left(\varphi_{\mathrm{v}}{ }^{0}\right)$ limiting apparent molar adiabatic compressibility $\left(\varphi_{\mathrm{k}}{ }^{0}\right)$ hydration number $(\mathrm{nH})$ and limiting apparent molar expansibility $\left(\varphi_{E}{ }^{0}\right)$ are calculated. The $\varphi_{v}$ values increase with an increase in temperature and concentration of urea and thiourea. 
The $\varphi_{v}$ values of urea become more negative than thiourea, indicating that strong hydrophilic hydration of urea occurs together with the clustering of water in the bulk. Due to the strong hydrophilic hydration (strong hydrogen bond) of urea, $\varphi_{k}$ values of urea increase than thiourea molecule. The $\varphi_{v}{ }^{0}$ values increase with an increase in temperature for both urea and thiourea. Solute-solvent interactions are more efficient for thiourea than urea because of more ability detachment of water molecule from thiourea. The $\varphi_{k}{ }^{0}$ values increase with an increase in temperature for both urea and thiourea. Because of more water molecule detachment from hydration sphere of thiourea, possibility of monomeric water molecule increases and $\varphi_{k}{ }^{0}$ values increase than urea molecule. The $\mathrm{nH}$ values of urea and thiourea decrease with an increase in temp. \& concentration of urea and thiourea. Due to the strong solute-solute interaction, more water molecule relaxes from hydration sphere of solute for both cases of temperature and concentration. More hydration number of thiourea are higher than urea because of increasing hydrophobic character for thiourea. From the expansibility measurement, it clearly observe that $\left(\partial^{2} \varphi_{v}{ }^{0} / \partial T_{2}\right)_{p}$ value for thiourea is more negative than urea solution. So, thiourea shows more structure breaking behavior than urea in solution.

\section{ACKNOWLEDGMENTS}

Authors are thankful to the Department of Chemistry, University of Rajshahi, Bangladesh for providing laboratory facilities to carry out this work.

\section{REFERENCES}

1. Hammes, G.; Schimmel, P. J. Am. Chem., 1967, 6028 (1960), 442-446.

2. Frank, H. S.; Franks, F. The J. of Chemical Physics., 1968, 48(10), 4746-4757.

3. Abu-Hamdiyyah, M. J. Phys. Chem., 1965, 69, 2720-2725.

4. Subramanian, S.; Balasubramanian, D.; Ahluwalia, J. C. J. Phys. Chem., 1969, 73 (1), 266-269.

5. Khatun, R. and Islam, N., Orient. J. Chem., 2012, 28(1), 165-187.

6. Motin, M. a.; Biswas, T. K.; Huque, E. M. Phys. Chem. Liq., 2002, 40, 593-605.

7. Krakowiak, J.;Wawer, J. J. Chem. Thermodyn., 2014, 79, 109-117.

8. Tarlok, S. B.; Aashima, B.; Navalpreet, K. and Parampaul K. B. J. of Chem. \& Eng. Data ., 2017, 62 (1), 20-34.

9. Khatun, M. R.; Islam, M. M.; Rima, F. R.; Islam, M. N. J. Chem. Eng. Data., 2016, 61 (1), 102-113.

10. Doyel, M. B.; Sudhakar, S. D. and Sangesh,
P. Z. The J. of Chem Therm., 2016, 101, 207220.

11. Latha, M.; Vijay, M. T.; Sudhakar, S. D.; Dinesh, W. D.; Santosh, P. J. The J. of Chem. Therm., 2017, 112, 166-177.

12. Burakowski, A.; Gli, J. Hydration Numbers of Nonelectrolytes from Acoustic Methods. Chem. Rev., 2012, 112, 2059-2081.

13. Rima, F. R.; Islam, M. M.; Islam, M. N. J. Chem. Eng. Data., 2013, 58 (11), 2991-2997.

14. Hakin, A. W.; Copeland, A. K.; Liu, J. L.; Marriott, R. A.; Preuss, K. E. Densities, J. Chem. Eng. Data., 1997, 42(1), 84-89.

15. Li, Y.; Li, Y.; Wang, F.; Ren, B. J. Chem. Thermodyn., 2013, 66, 14-21.

16. Pal, A.; Chauhan, N. J. Chem. Thermodyn., 2012, 54, 288-292.

17. Hepler, L. G. Can. J. Chem., 1969, 47(24), 4613-4617.

18. Kabir, M. H.; Nath, R. K.; Hossain, M. K.; Hyder, M. K. M. Z., Orient. J. Chem., 2018, 34(1), 196-202 